\title{
Batch-to-batch N-glycosylation study of infliximab, trastuzumab and bevacizumab, and stability study of bevacizumab
}

\author{
Ana Planinc, ${ }^{1,2}$ Bieke Dejaegher, ${ }^{2,3,4}$ Yvan Vander Heyden, ${ }^{2,4}$ Johan Viaene, ${ }^{2,4}$ \\ Serge Van Praet, ${ }^{5}$ Florence Rappez, ${ }^{5}$ Pierre Van Antwerpen, ${ }^{1,2}$ Cédric Delporte ${ }^{1,2}$
}

\begin{abstract}
- Additional material is published online only. To view please visit the journal online (http://dx.doi.org/ 10.1136/ejhpharm-2016001022)

${ }^{1}$ Analytical Platform of the Faculty of Pharmacy and Laboratory of Pharmaceutical Chemistry, Faculty of Pharmacy, Université Libre de Bruxelles (ULB), Brussels, Belgium

${ }^{2}$ Joint Research Group ULBVUB, Brussels, Belgium ${ }^{3}$ Laboratory of Instrumental Analysis and Bioelectrochemistry, Faculty of Pharmacy, Université Libre de Bruxelles (ULB), Brussels, Belgium

${ }^{4}$ Department of Analytical Chemistry and Pharmaceutical Technology (FABI), Faculty of Medicine and Pharmacy, Center for Pharmaceutical Research (CePhaR), Vrije Universiteit Brussel (VUB), Brussels, Belgium ${ }^{5}$ Pharmacy, CHU Saint-Pierre, Brussels, Belgium
\end{abstract}

\section{Correspondence to} Ana Planinc, Faculty of Pharmacy, Laboratory of Pharmaceutical Chemistry and Analytical Platform of the Faculty of Pharmacy, Université Libre de Bruxelles (ULB), Bld du Triomphe, Campus Plaine, CP 205/05B, Brussels B-1050, Belgium; aplaninc@ulb.ac.be

Received 15 June 2016 Revised 24 August 2016 Accepted 30 August 2016 Published Online First 15 September 2016

EAHP Statement 5: Patient Safety and Quality Assurance

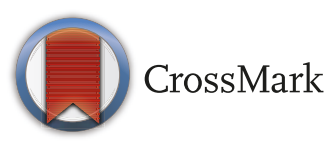

To cite: Planinc $A$, Dejaegher B, Vander Heyden Y, et al. Eur J Hosp Pharm 2017;24:286-292.

\begin{abstract}
Objectives Infliximab, trastuzumab and bevacizumab are among the most frequently prescribed therapeutic proteins, and like most other therapeutic proteins, are glycosylated. As differences in glycosylation may significantly change the safety and efficacy of therapeutic glycoproteins, it is extremely important to control $\mathrm{N}$-glycosylation consistency. In the first part of this study, the batch-to-batch consistency of the N-glycosylation of infliximab, trastuzumab and bevacizumab was analysed. In the second part, the consistency of the N-glycosylation of bevacizumab stored in polycarbonate syringes (for offlabel drug use) for 3 months was examined.
\end{abstract}

Methods N-glycans were (i) enzymatically released

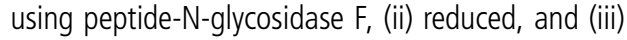
analysed using hydrophilic interaction liquid chromatography coupled with high-resolution mass spectrometry. Mass spectrometry data were interpreted using principal component analysis combined with twoway analysis of variance and Tukey post hoc tests. The biological activity of infliximab and trastuzumab was examined using enzyme-linked immunosorbent assays.

Results The results of both studies make important contributions to the field of hospital pharmacy. All batches of the studied therapeutic glycoproteins (infliximab, trastuzumab and bevacizumab) varied considerably (especially in galactosylation), while the $\mathrm{N}$-glycosylation of bevacizumab remained unchanged during 3-month storage.

Conclusions Threshold values for batch-to-batch $\mathrm{N}$-glycosylation variations should be established and batch-to-batch glycosylation consistency should be regularly tested. In our study, samples with significantly different N-glycosylation profiles showed no significant variations in biological activity, suggesting that the differences are probably not therapeutically significant.

\section{INTRODUCTION}

Therapeutic proteins are growing in importance due to their efficacy for treating diseases such as cancers, immune disorders and infections. ${ }^{12}$ The majority of therapeutic proteins are glycosylated and even minor changes in the structure of the attached glycans can have a crucial impact on their safety, efficacy and quality, highlighting the importance of $\mathrm{N}$-glycosylation quality control. ${ }^{3-5}$

In the present study, we investigated the $\mathrm{N}$-glycans of three widely used therapeutic glycoproteins: infliximab, trastuzumab and bevacizumab. Infliximab is a chimeric monoclonal antibody targeting tumour necrosis factor $\alpha(\mathrm{TNF}-\alpha)$ and is used in the treatment of autoimmune diseases, such as Crohn's disease, rheumatoid arthritis and psoriasis. ${ }^{6}$ Infliximab is the most extensively studied therapeutic glycoprotein in the context of biosimilar products. ${ }^{7}$ Trastuzumab is a recombinant humanised monoclonal antibody binding the HER2/neu receptor and is used in the treatment of certain breast cancers. ${ }^{8}$ Finally, bevacizumab is a recombinant humanised monoclonal antibody against vascular endothelial growth factor, and is used in the treatment of metastatic colorectal cancer as well as ovary, breast, lung and kidney cancer. Bevacizumab is also used off-label to treat neovascular age-related macular degeneration. ${ }^{9}$

$\mathrm{N}$-glycans released from infliximab, trastuzumab and bevacizumab have different structures. In general, $\mathrm{N}$-glycans are classified into three groups: high mannose glycans, complex glycans and hybrid glycans (see figure 1). The high mannose glycans contain several mannose units attached to $\mathrm{N}$-acetylglucosamine. The complex glycans are usually terminally galactosylated, terminally sialylated and/or core-fucosylated. ${ }^{10}$ The hybrid glycans have some high mannose glycans features and some complex glycans features. In this article, the Oxford Glycobiology Institute (University of Oxford) notation has been used (see figure 1) to describe the monosaccharide sequences and linkages. ${ }^{11}$

It is extremely challenging for the biopharmaceutical industry to obtain consistent $\mathrm{N}$-glycosylation profiles since glycosylation is the most complex post-translational modification. As N-glycosylation consistency ensures the efficacy and safety of therapeutic glycoproteins, ${ }^{4}{ }^{12}$ the $\mathrm{N}$-glycosylation batch-to-batch consistency has to be regularly tested.

The first aim of our study was to examine whether there were differences in the $\mathrm{N}$-glycosylation profiles of several batches of infliximab (12 batches), trastuzumab (9 batches) and bevacizumab (7 batches). Our second aim was to monitor the $\mathrm{N}$-glycosylation stability of reconstituted bevacizumab solution over 12 weeks as N-glycosylation may change during storage. Hospital pharmacies prepare bevacizumab solutions for injection in ready-to-use syringes for the treatment of age-related macular degeneration (offlabel drug use). Recently, an extensive study conducted to assess the stability of bevacizumab packed in plastic syringes found the primary, secondary and tertiary structures of bevacizumab were unchanged. ${ }^{13}$ In contrast, another study claimed that the activity of bevacizumab stored in plastic syringes may slightly degrade over time. ${ }^{14}$ However, the stability of the 


\section{High mannose glycans}

M3<smiles>OOOOO</smiles>

Complex glycans
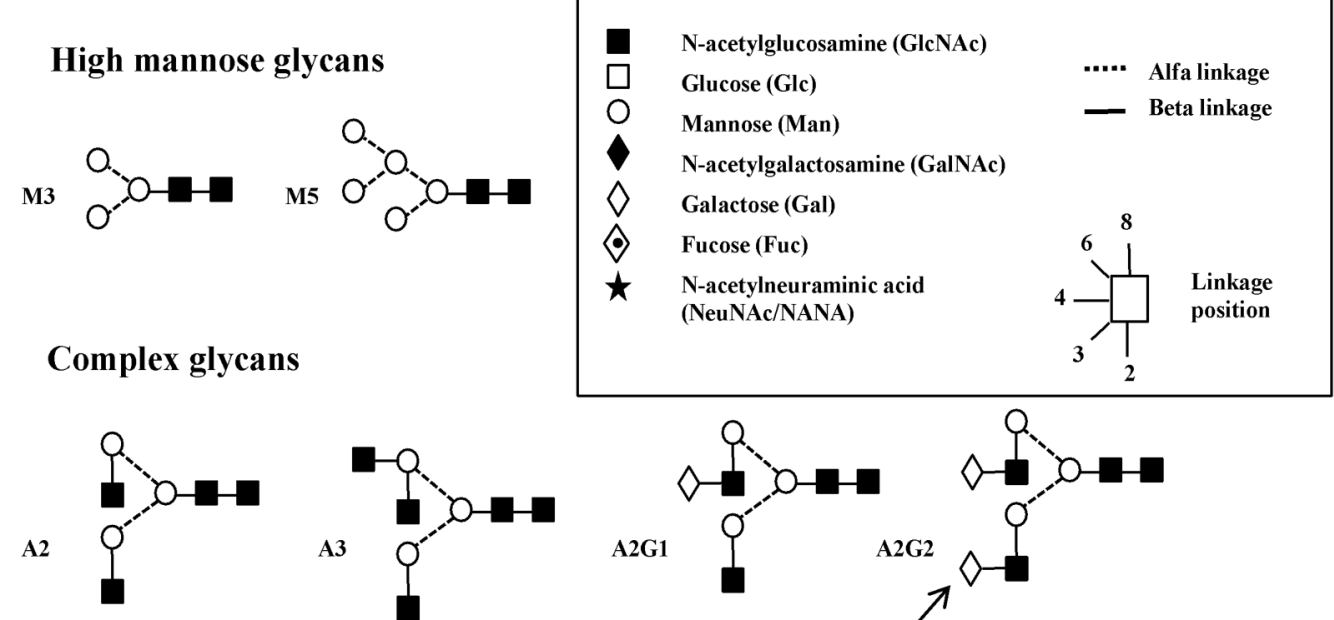

A3

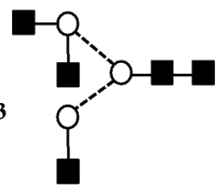

A2G1

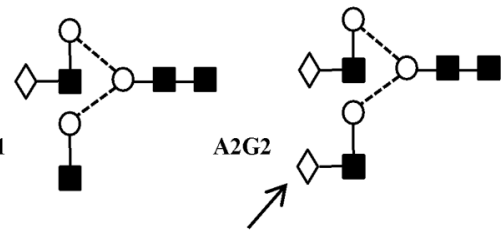

Galactosylated glycans

Core-fucosylated glycans

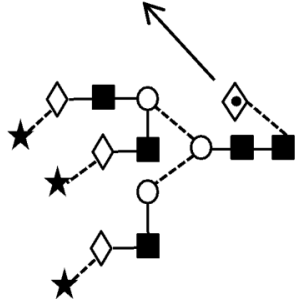

FM5A1G

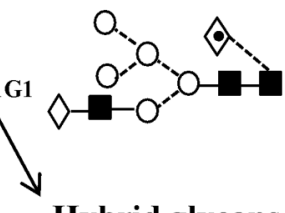

Hybrid glycans

Sialylated glycans

Figure 1 Schematic representation of N-glycans using the Oxford Glycobiology Institute (University of Oxford) notation, showing the structures of high mannose glycans, complex glycans and hybrid glycans.

$\mathrm{N}$-glycosylation of bevacizumab during storage has not yet been comprehensively studied.

\section{MATERIALS AND METHODS \\ Reagents and chemicals}

Sodium phosphate, sodium dodecyl sulfate (SDS), potassium hydroxide, monosodium phosphate dihydrate and potassium chloride were obtained from Merck (Darmstadt, Germany). Ammonium acetate, 2-mercaptoethanol, sodium borohydride, PNGase F (from Elizabethkingia meningoseptica, P7367) and trifluoroacetic acid were purchased from Sigma-Aldrich (Steinheim, Germany). Acetic acid and ammonium formate were obtained from VWR International (Leuven, Belgium), acetonitrile and methanol from Fluka (Steinheim, Germany), Nonidet P40 from Roche Diagnostics (Vilvoorde, Belgium), Nanosep 10K omega spin filters from Pall Corporation (New York, USA) and PGC-SPE columns from Supelco (Bellefonte, Pennsylvania, USA). The pharmacy of Saint-Pierre University Hospital (Brussels, Belgium) provided samples of the approved drugs infliximab, trastuzumab and bevacizumab prepared as mentioned in the Summary of Product Characteristics (SPC).

\section{Sample preparation}

In the present study, the $\mathrm{N}$-glycosylation of 12 batches of infliximab, nine batches of trastuzumab and seven batches of bevacizumab was analysed. Each product was prepared as recommended by the manufacturer. For the bevacizumab stability study, the drug was transferred under aseptic conditions from the original vial into syringes (1 mL Luer Lok, TDS-309628) obtained from BD (Rolle, Switzerland). The barrels and plunger rods of the syringes are made of polycarbonate, the stoppers of latex-free elastomer, and the lubricant of medical grade silicone oil. The bevacizumab syringes were stored at $4^{\circ} \mathrm{C}$ for 3 months. Each week, one syringe was withdrawn as a sample and frozen at $-80^{\circ} \mathrm{C}$ until preparation of $\mathrm{N}$-glycans for analysis. The stability study experiments were repeated three times over 3 months.

The method for glycan preparation from proteins was adapted from our previously established protocol. ${ }^{15}$ Briefly, each glycoprotein sample (equivalent to $1 \mathrm{mg}$ protein) was first denatured using SDS, 2-mercaptoethanol and Nonidet P40. PNGase $\mathrm{F}$ was then added to release $\mathrm{N}$-glycans. Proteins were removed by centrifugation and using Nanosep 10K omega spin filters. $\mathrm{N}$-glycans were then reduced by sodium borohydride in $\mathrm{KOH}$. Samples were cleaned using porous graphitised carbon cartridges (100 mg, $1 \mathrm{~mL}$ tubes). Dried samples were dissolved in $50 \mu \mathrm{L}$ of water/acetonitrile $(50 / 50, \mathrm{v} / \mathrm{v})$ before injection on to an LC-MS system.

\section{HILIC coupled to electrospray ionisation-MS}

The method for the separation and detection of $\mathrm{N}$-glycans was adapted from our previously established protocol. ${ }^{15}$ Briefly, analyses were performed using a 1200 series rapid resolution liquid chromatograph coupled to a 6520 series electrospray ionisation (ESI)-quadrupole time-of-flight (QTOF) mass spectrometer from Agilent Technologies (Waldbronn, Germany). Separation of the glycans was performed using an XBridge BEH Amide column (130 ̊, $2.5 \mu \mathrm{m}, 150 \mathrm{~mm} \times 2.1 \mathrm{~mm}$ ID) obtained from Waters (Zellik, Belgium). Data acquisition and data analysis were carried out using MassHunter Acquisition software for QTOF (V.B.04 SP3) and MassHunter Qualitative Analysis (V.B.06) software (both from Agilent Technologies). 


\section{ELISA procedure}

The binding of infliximab and trastuzumab to their targets was tested using an Infliximab ELISA Kit (ABIN2862648) and a Trastuzumab ELISA Kit (ABIN1540259), both from Anticorps (Aachen, Germany). Infliximab (batches 5 and 12) and trastuzumab (batches 5 and 6) samples were diluted 10000 times before the manufacturer's instructions were followed.

\section{Data handling}

Data pre-processing

Before data analysis, the data were organised in an $n \times p$ data matrix $X(n=$ number of samples and $p=$ number of $\mathrm{N}$-glycans). The relative abundance of a given $\mathrm{N}$-glycan is presented for each matrix cell. Depending on the examined data, the relative abundances of some or all $\mathrm{N}$-glycans (using integrated areas under the curve for related peaks) were considered as variables. M5, FA2, FA2G1, FA2G2, A2, A2G1, A1, FA1, FA1G1 and FM5A1G1 were considered as variables for infliximab; M5, M6, M7, M8, A1, A2, A2G1, A2G2, FA1, FA2, FA1G1, FA2G1, FA2G2, FA2G2S1 and FA2G2S2 for trastuzumab; and FA2, FA2G1, FA2G2, A2, A1, FA1, FA1G1 and M5 for bevacizumab. No pre-processing was performed.

\section{Principal component analysis}

As previously described, ${ }^{15}$ principal component analysis (PCA) was applied to determine whether it was possible to identify differences in the $\mathrm{N}$-glycosylation profile between the infliximab, trastuzumab and bevacizumab batches, and between different weeks in the bevacizumab stability study.

Two-way analysis of variance and Tukey post hoc test Two-way analysis of variance (ANOVA) ${ }^{16}{ }^{17}$ was applied to analyse whether there were differences in the N-glycosylation of the therapeutic glycoproteins between batches or between weeks (stability study). When a difference was observed, a Tukey ${ }^{17}$ post hoc test was performed to determine which batches or weeks (stability study) differed. In all analyses, $\alpha$ was set at 0.05 and $\mathrm{p}$ values $\leq 0.05$ were considered significant.
T-test

A t-test ${ }^{18}$ was applied to analyse whether there were differences between the infliximab batches (batches 5 and 12) and trastuzumab batches (batches 5 and 6) in binding to their targets. p Values $\leq 0.05$ were considered significant.

\section{Software}

PCA was performed using Past 3.x (Windows, zipped exe, V.3.11). Two-way ANOVA, Tukey post hoc tests and t-tests were performed using a trial version of IBM SPSS Statistics V.22.

\section{RESULTS}

\section{Infliximab batch-to-batch study}

Twelve different batches of infliximab were analysed. Three replicates of each batch were prepared and each sample was injected three times on to the LC-MS system. The relative abundances of major N-glycans (M5, FA2, FA2G1, FA2G2, A2, A2G1, A1, FA1, FA1G1 and FM5A1G1) were compiled.

PCA was performed on the infliximab data (108 observations $\times 10$ N-glycans as variables). Separation along PC1 $(92.25 \%)$ was mainly caused by variables FA2G1, FA2 and FA1, while separation along PC2 (6.50\%) was mainly caused by FA2, FA1 and M5, as determined by loading plots (not shown). Two groups of infliximab batches were revealed by the PC1-PC2 score plot (figure 2) with batches 1, 2, 7, 8, 910 and 12 forming one cloud (first group) and batches 3, 4, 5, 6 and 11 forming another cloud (second group).

The data revealed that batches $1,2,7,8,9,10$ and 12 contained a higher relative abundance of complex N-glycans (galactosylated: FA2G1 and FA2G2) and lower amounts of simple $\mathrm{N}$-glycans (M5, A1 and A1G1). The amount of FA2 N-glycan varied between the batches by about $8 \%$ (see online supplementary table S1).

There was a statistically significant difference between batches for all main $\mathrm{N}$-glycans as determined by two-way ANOVA $(p \leq 0.001)$. One batch from the first group (batch 5) and one batch from the second group (batch 12) were selected and statistically compared. A Tukey post hoc test comparing batches 5 and 12 revealed that the relative abundances of FA2G1, FA2G2,
Figure 2 PC1-PC2 score plot of 12 batches of infliximab: data profile of $10 \mathrm{~N}$-glycans from 108 observations of infliximab ( 12 batches $\times 3$ replicates $\times 3$ injections). The differences between the 12 batches of infliximab are indicated. No pre-processing was performed. The $95 \%$ confidence ellipses are marked for each class.

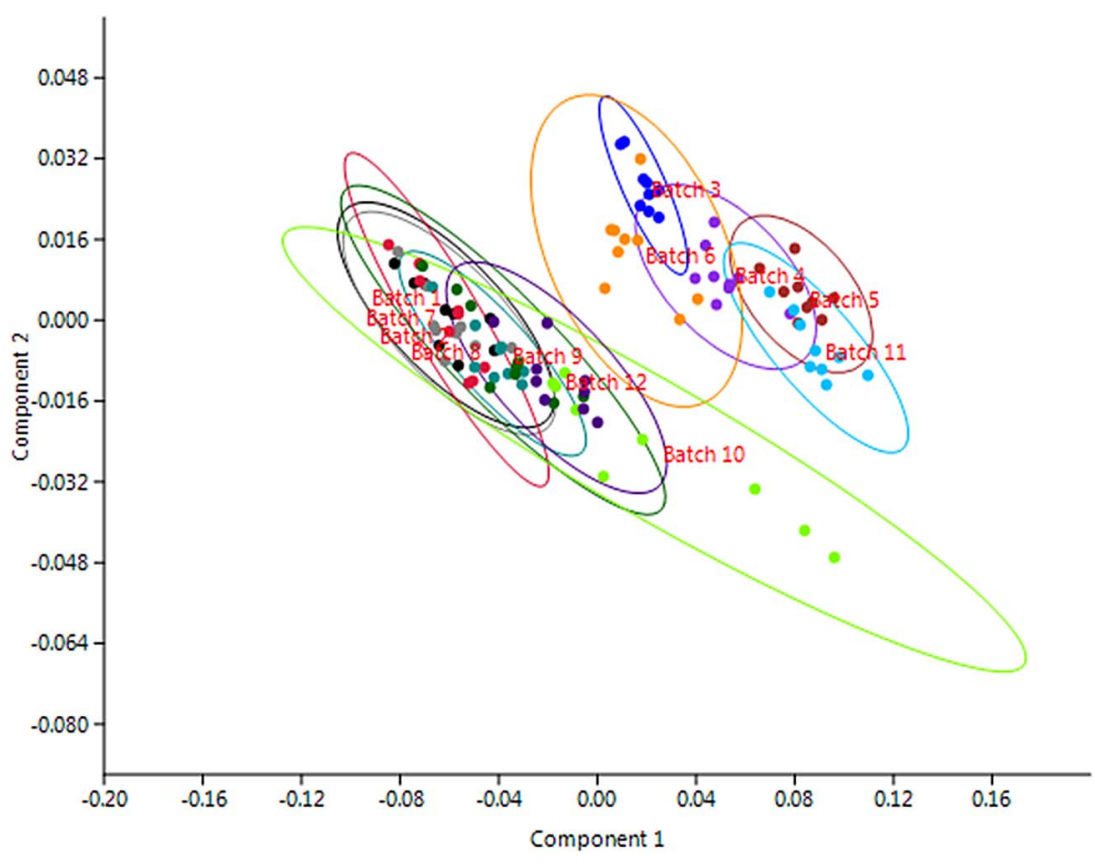


M5, A1 and A1G1 N-glycans all differed significantly $(p \leq 0.001)$. These statistical results confirmed our predictions obtained by observing the PC1-PC2 score plot and raw data.

\section{Trastuzumab batch-to-batch study}

Nine trastuzumab batches were analysed. Three replicates of each batch were prepared and each sample was injected three times on to the LC-MS system. The relative abundances of major N-glycans (M5, M6, M7, M8, A1, A2, A2G1, A2G2, FA1, FA2, FA1G1, FA2G1, FA2G2, FA2G2S1 and FA2G2S2) were compiled.

PCA was performed on the trastuzumab data (81 observations $\times 15 \mathrm{~N}$-glycans as variables). Separation along PC1 $(84.24 \%)$ was mainly caused by variables FA2, FA2G1 and FA2G2, while separation along PC2 (10.79\%) was mainly caused by FA2G1 and M5, as determined by loading plots (not shown). The PC1-PC2 score plot (figure 3) indicated that the trastuzumab batches varied. All batches formed individual clouds with batch 6 varying the most along PC1.

Raw data showed that batch 6 differed regarding the $\mathrm{N}$-glycans FA2, FA2G1 and FA2G2 and that it contained more FA2 N-glycan and therefore less FA2G1 and FA2G2 N-glycans. The data also revealed that the most variable $\mathrm{N}$-glycans were FA2 (variation between batches of around 8\%) and FA2G1 (variation between batches of around 5\%) (see online supplementary table S2).

Two-way ANOVA showed that there was a statistically significant difference between batches for all main $\mathrm{N}$-glycans $(\mathrm{p} \leq 0.001)$. Batches 6 and 5 were selected and statistically compared. The Tukey post hoc test revealed that the relative abundances of FA2, FA2G1 and FA2G2 N-glycans all differed significantly $(\mathrm{p}<0.001)$. These statistical results confirmed our predictions obtained by observing the PC1-PC2 score plot and raw data.

\section{Bevacizumab batch-to-batch study}

Seven batches of bevacizumab were analysed. Three replicates of each batch were prepared and each sample was injected three times on to the LC-MS system. The relative abundances of major N-glycans (FA2, FA2G1, FA2G2, A2, A1, FA1, FA1G1 and M5) were compiled.
PCA was performed on the bevacizumab data (63 observations $\times 8 \quad \mathrm{~N}$-glycans as variables). Separation along PC1 $(88.94 \%)$ was mainly caused by the variables FA2, FA2G1 and FA2G2, while separation along PC2 (9.49\%) was mainly caused by FA2G1, FA2G2 and FA2, as determined by loading plots (not shown). The PC1-PC2 score plot (figure 4) of the seven batches revealed that the bevacizumab batches varied.

Raw data showed that batch 7 contained more FA2 N-glycan and less FA2G1 and FA2G2 N-glycans. Online supplementary table $\mathrm{S} 3$ reveals that the most variable $\mathrm{N}$-glycans were FA2 (variation between batches of around 8\%) and FA2G1 (variation between batches of around 5\%).

In order to confirm the PCA and raw data observations, two-way ANOVA and Tukey post hoc tests were conducted. There was a statistically significant difference between batches for all the main $\mathrm{N}$-glycans $(\mathrm{p} \leq 0.001)$. Batches 7 and 3 were then selected and statistically compared. The Tukey post hoc test revealed that the relative abundances of FA2, FA2G1 and FA2G2 $\mathrm{N}$-glycans differed significantly ( $\mathrm{p} \leq 0.008)$. Again, these statistical results confirmed our findings from the PCA and raw data.

\section{Bevacizumab stability study}

We tested the $\mathrm{N}$-glycosylation stability of the bevacizumab stored in syringes for 3 months. The relative abundances of major N-glycans (FA2, FA2G1, FA2G2, A2, A1, FA1, FA1G1 and M5) were compiled.

PCA was performed on the bevacizumab data (117 observations $\times 8 \mathrm{~N}$-glycans as variables). Separation along PC1 $(92.02 \%)$ was mainly caused by the variables FA2 and FA2G1, while separation along PC2 (4.80\%) was mainly caused by FA2G1, FA2G2 and FA2, as determined by loading plots (not shown). From the PC1-PC2 score plot (figure 5), it can be seen that N-glycosylation of bevacizumab remained unchanged during the studied period. Raw data confirmed that $\mathrm{N}$-glycosylation of bevacizumab remained the same during the 12 weeks (see online supplementary table S4).

To confirm that N-glycosylation of bevacizumab remains unchanged, we performed two-way ANOVA and post hoc tests (Tukey tests). The Tukey post hoc test revealed that there was no statistically significant difference between the weeks for all main $\mathrm{N}$-glycans ( $\mathrm{p}>0.05)$, except for some $\mathrm{N}$-glycans in week 10 .
Figure 3 PC1-PC2 score plot of nine batches of trastuzumab: data profile of $15 \mathrm{~N}$-glycans from 91 observations of trastuzumab ( 9 batches $\times 3$ replicates $\times 3$ injections). The differences between nine batches of trastuzumab are indicated. No pre-processing was performed. The $95 \%$ confidence ellipses are marked for each class.

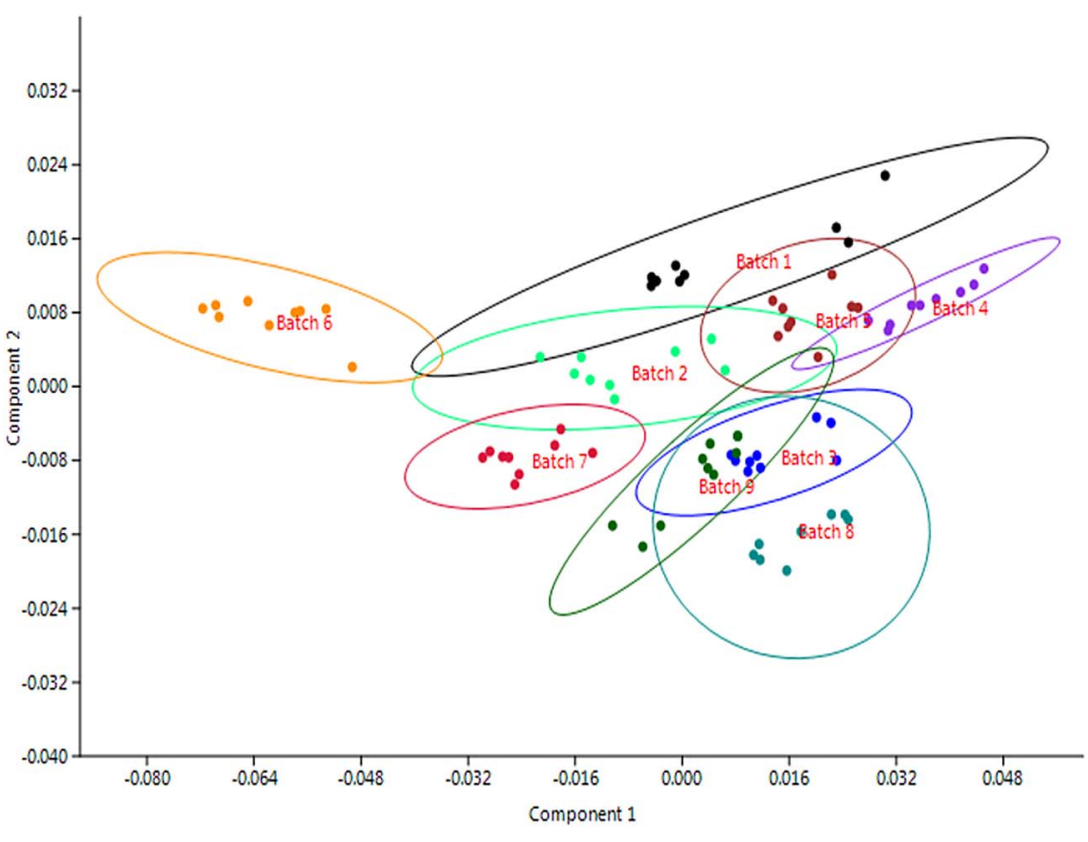




\section{Original article}

Figure $4 \quad P C 1-P C 2$ score plot of seven batches of bevacizumab: data profile of $8 \mathrm{~N}$-glycans from 63 observations of bevacizumab (7 batches $\times 3$ replicates $\times 3$ injections). The differences between the seven batches of bevacizumab are indicated. No pre-processing was performed. The 95\% confidence ellipses are marked for each class.
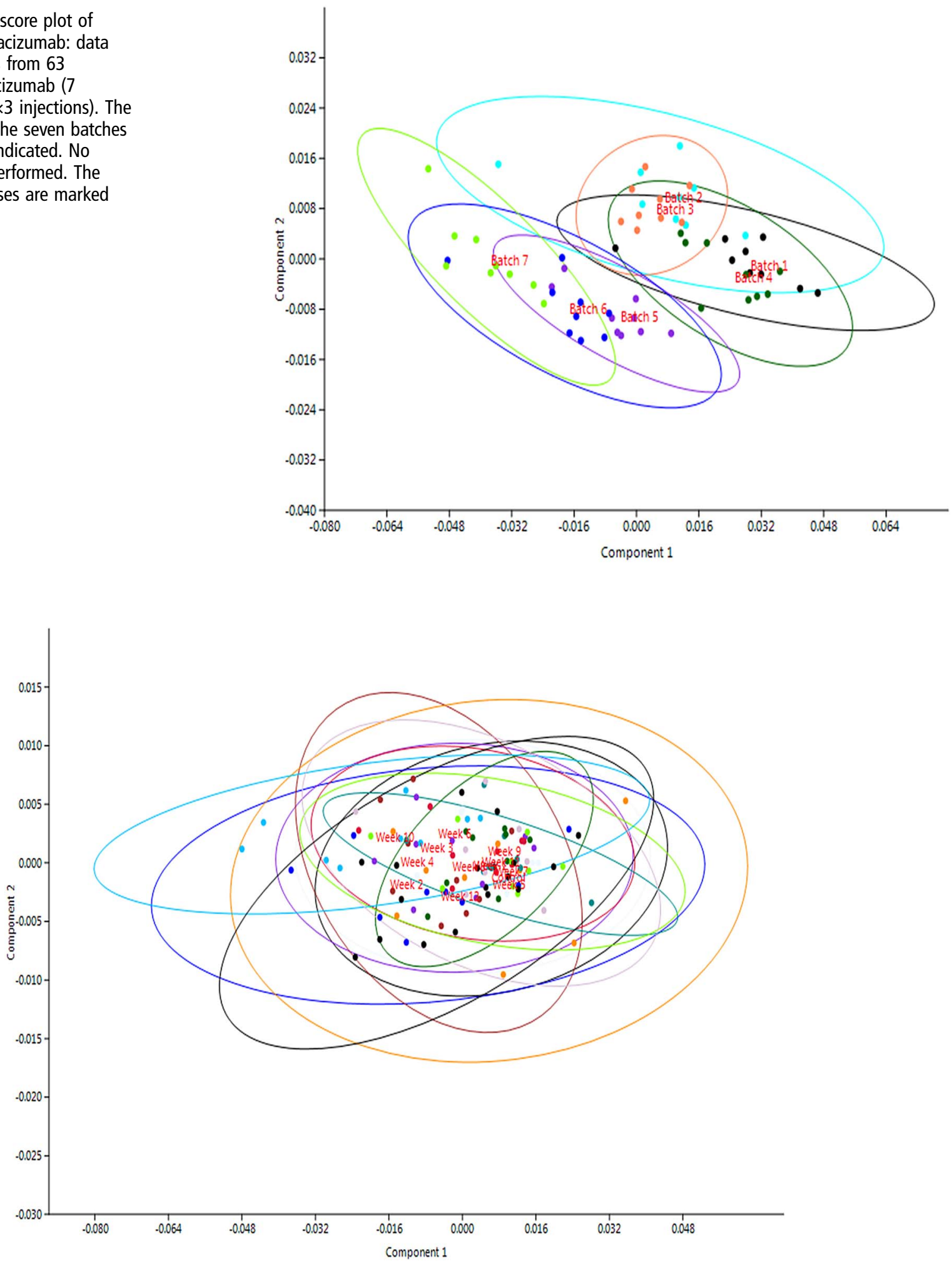

Figure 5 PC1-PC2 score plot of the stability study of bevacizumab: data profile of $8 \mathrm{~N}$-glycans from 117 observations of bevacizumab (control and 12 weeks $\times 3$ replicates $\times 3$ injections). The differences between the 12 weeks of storage at $4^{\circ} \mathrm{C}$ are indicated. No pre-processing was performed. The $95 \%$ confidence ellipses are marked for each class.

However, we can conclude that N-glycosylation of bevacizumab does not change over a 12 -week period.

\section{ELISA test for infliximab and trastuzumab}

In the present study, as significant differences in N-glycosylation between some batches were found, we investigated whether those differences affect antibody binding to antigens. Two statistically different batches of infliximab (batches 5 and 12) and two statistically different batches of trastuzumab (batches 5 and 6) were selected and binding to their two targets (TNF- $\alpha$ and HER2/neu receptor) was examined using an Infliximab ELISA Kit and a Trastuzumab ELISA Kit, respectively. Results showed 
that samples with significantly different $\mathrm{N}$-glycosylation patterns still bind their targets successfully. A t-test revealed that infliximab batches 5 and 12 do not differ significantly $(p=0.8693$, $\mathrm{n}=5$ ) and that trastuzumab batches 5 and 6 do not differ significantly $(\mathrm{p}=0.1022, \mathrm{n}=5)$ (figure 6).

\section{DISCUSSION}

Some earlier studies examining the batch-to-batch $\mathrm{N}$-glycosylation consistency of infliximab ${ }^{19}$ and trastuzumab ${ }^{20} 21$ have been identified but no extensive studies on bevacizumab have been found. In the infliximab study of three batches, the authors detected minor batch-to-batch variability in M5, A2 and FA2G1 N-glycans. ${ }^{19}$ In the two studies investigating trastuzumab, the authors identified significant glycosylation differences between batches. One study compared three batches of trastuzu$\mathrm{mab}^{20}$ and the other four batches. ${ }^{21}$ However, a comparison of three or four batches may be too small to determine consistency between batches. Consequently, in our study we examined a larger number of batches (12 of infliximab, nine of trastuzumab and seven of bevacizumab) and also analysed the binding efficiency of two batches of trastuzumab and two batches of infliximab to determine if differences in N-glycosylation between batches affect biological activity.

Results showed that N-glycosylation varies between infliximab, trastuzumab and bevacizumab batches. The $\mathrm{N}$-glycans showing the most variability are FA2, FA2G1 and FA2G2, which means that batches differ in terminal galactosylation. These results suggest that some batches might undergo poorer synthesis of complex (galactosylated) N-glycans during production.

The N-glycan variability between the trastuzumab and bevacizumab batches can be explained by the fact that these therapeutic proteins are produced in Chinese hamster ovary (CHO) cells. $\mathrm{CHO}$ cells are a preferred host cell line due to sufficient
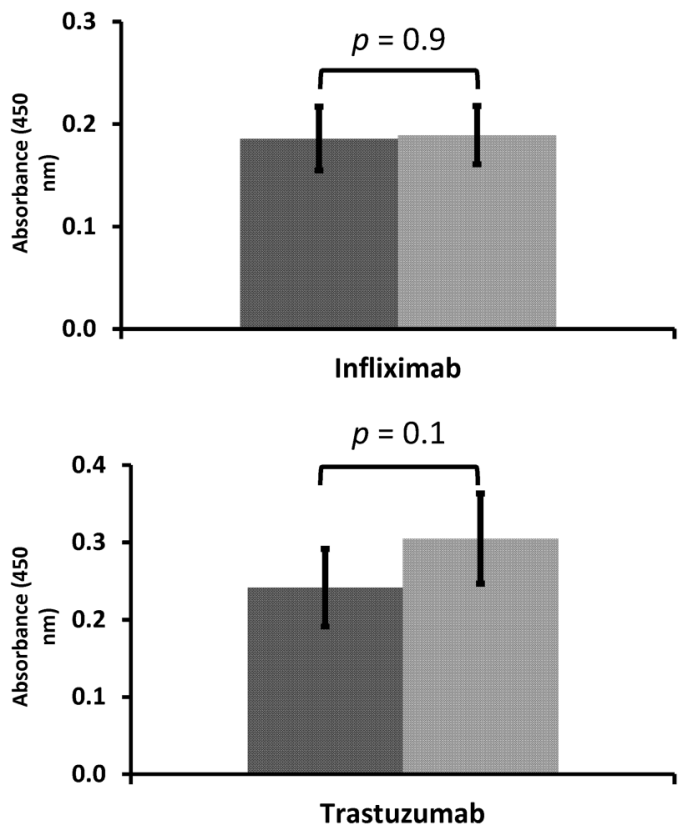

Figure 6 Infliximab and trastuzumab ELISA tests: two batches of infliximab and two batches of trastuzumab with significantly different $\mathrm{N}$-glycosylation were compared using the ELISA activity test. A t-test revealed that infliximab batches 5 and 12 do not differ significantly $(p=0.8693, n=5)$ and that trastuzumab batches 5 and 6 do not differ significantly $(p=0.1022, n=5)$. expression of the sialylated N-glycans. Nevertheless, the main disadvantage of $\mathrm{CHO}$ mammalian cells is that glycosylation is not constant as hundreds of enzymes are involved in the glycosylation process. ${ }^{22}$ This results in different glycosylation patterns, as observed in our study.

Regarding batch-to-batch differences in therapeutic glycoproteins, the European Medicines Agency only reports that 'Minor differences among the materials investigated were identified in C-terminal heterogeneity, glycosylation pattern and charge heterogeneity but studies performed showed that these differences are unlikely to affect the safety and efficacy of the product'. ${ }^{23}$ From our study, it is evident that $\mathrm{N}$-glycosylation varies by up to $8 \%$ for individual $\mathrm{N}$-glycans, so we wondered whether $\mathrm{N}$-glycosylation differences affect the safety and efficacy of therapeutic glycoproteins. Therefore, we used ELISA kits to examine how successful infliximab and trastuzumab were in binding to their targets. Samples with significantly different $\mathrm{N}$-glycosylation both bound their targets successfully with no statistical differences, suggesting that the N-glycosylation variations between the batches (shown in our study) could be acceptable and are in accordance with biological variability. However, further studies are recommended to confirm this and to determine if safety is affected.

To conclude, differences between 12 batches of infliximab, nine batches of trastuzumab and seven batches of bevacizumab were studied as changes in glycosylation profiles influence the safety, efficacy and quality of therapeutic glycoproteins. ${ }^{5}$ For example, changes in terminal galactosylation and/or sialylation affect the half-life and the efficacy (anti-inflammatory effect) of therapeutic glycoproteins, ${ }^{24}$ changes in core fucosylation may alter their anti-inflammatory effects, ${ }^{25}$ and changes in mannosylation can affect their half-life and cause off-target hepatic toxicity. ${ }^{26}$ Pharmaceutical companies must continue to monitor $\mathrm{N}$-glycosylation batch-to-batch consistency, as N-glycosylation can be significantly changed by slightly altered culture conditions during production. Pharmaceutical companies should also establish threshold values for $\mathrm{N}$-glycosylation variations between batches as they have not yet been specified. Moreover, batch-to-batch studies should always be conducted on a sufficient number of batches.

The N-glycosylation stability study of bevacizumab samples transferred from vials into plastic syringes and refrigerated for 3 months,

\section{What this paper adds}

What is already known on this subject

- Previous batch-to-batch N-glycosylation consistency studies of infliximab and trastuzumab revealed minor $\mathrm{N}$-glycosylation batch-to-batch variability.

- However, those studies were conducted on only three or four batches, which is certainly not enough.

What this study adds

- Three extensive $\mathrm{N}$-glycosylation consistency studies of infliximab, trastuzumab and bevacizumab showed that batches vary considerably (especially in galactosylation), although there were no significant differences in biological activity.

- An N-glycosylation stability study of bevacizumab stored in polycarbonate syringes showed that $\mathrm{N}$-glycosylation remained unchanged over 3 months. 
shows that $\mathrm{N}$-glycosylation of bevacizumab remains unchanged during 3-month storage. This is important for the quality of the product as are other factors including biological activity, purity and detection of the conjugated products. All important quality characteristics should be monitored in order to ensure the quality of biological products is maintained during storage.

Contributors AP conceived of the study and initiated study design, while PVA and CD helped with implementation. YVH, BD and JV provided statistical expertise. SVP and FR supplied the biopharmaceutical samples. All authors contributed to refining the study protocol and approved the final manuscript.

Funding This study was supported by grants from the Belgian Fund for Scientific Research (FRS-FNRS), no. 34553.08, a grant from the FER 2007 (ULB), and a grant from Slovenia (Ad futura). CD is a postdoctoral researcher funded by the FRS-FNRS.

Competing interests None declared.

Provenance and peer review Not commissioned; externally peer reviewed.

\section{REFERENCES}

1 Carter PJ. Introduction to current and future protein therapeutics: a protein engineering perspective. Exp Cell Res 2011;317:1261-9.

2 Walsh G. Biopharmaceutical benchmarks 2010. Nat Biotechnol 2010;28:917-24.

3 Planinc A, Bones J, Dejaegher B, et al. Glycan characterization of biopharmaceuticals: updates and perspectives. Anal Chim Acta 2016;921:13-27.

4 Hossler P, Khattak SF, Li ZJ. Optimal and consistent protein glycosylation in mammalian cell culture. Glycobiology 2009;19:936-49.

5 Delobel A, Cantais F, Catrain A, et al. Therapeutic antibody glycosylation analysis: a contract research organization perspective in the frame of batch release or comparability support. Methods Mol Biol 2013;988:115-43.

6 Raychaudhuri SP, Raychaudhuri SK. Biologics: target-specific treatment of systemic and cutaneous autoimmune diseases. Indian J Dermatol 2009:54:100-9.

7 McKeage K. A review of CT-P13: an infliximab biosimilar. BioDrugs 2014;28:313-21.

8 Sanchez-De Melo I, Grassi P, Ochoa F, et al. N-glycosylation profile analysis of trastuzumab biosimilar candidates by normal phase liquid chromatography and MALDI-TOF MS approaches. J Proteomics 2015;127:225-33

9 Ziemssen F, Grisanti S, Bartz-Schmidt KU, et al. Off-label use of bevacizumab for the treatment of age-related macular degeneration: what is the evidence? Drugs Aging 2009;26:295-320.

10 Raju TS, Briggs JB, Borge SM, et al. Species-specific variation in glycosylation of IgG: evidence for the species-specific sialylation and branch-specific galactosylation and importance for engineering recombinant glycoprotein therapeutics. Glycobiology 2000;10:477-86
11 Harvey DJ, Merry AH, Royle L, et al. Proposal for a standard system for drawing structural diagrams of $\mathrm{N}$ - and 0 -linked carbohydrates and related compounds. Proteomics 2009;9:3796-801.

12 Walsh $\mathrm{G}$, Jefferis R. Post-translational modifications in the context of therapeutic proteins. Nat Biotech 2006;24:1241-52.

13 Paul M, Vieillard V, Roumi E, et al. Long-term stability of bevacizumab repackaged in $1 \mathrm{~mL}$ polypropylene syringes for intravitreal administration. Ann Pharm Fr 2012;70:139-54.

14 Bakri SJ, Snyder MR, Pulido JS, et al. Six-month stability of bevacizumab (Avastin) binding to vascular endothelial growth factor after withdrawal into a syringe and refrigeration or freezing. Retina 2006;26:519-22.

15 Planinc A, Dejaegher B, Heyden YV, et al. LC-MS analysis combined with principa component analysis and soft independent modelling by class analogy for a better detection of changes in N-glycosylation profiles of therapeutic glycoproteins. Anal Bioanal Chem 2016.

16 Armstrong RA, Slade SV, Eperjesi F. An introduction to analysis of variance (ANOVA) with special reference to data from clinical experiments in optometry. Ophthalmic Physiol Opt 2000;20:235-41.

17 Kohout FJ, Norwood GJ. Interpretation of research data: analysis of variance. Am J Health Syst Pharm 1981;38:96-104.

18 Pillemer DB. One- versus two-tailed hypothesis tests in contemporary educational research. Educ Res 1991:20:13-17.

19 Waters Corporation. Structural comparison of infliximab and a biosimilar via subunit analysis using the biopharmaceutical platform with UNIFI. http://www.waters.com/ webassets/cms/library/docs/720004796en.pdf (accessed 13 May 2016).

20 Waters Corporation. Trastuzumab Glycan batch-to-batch profiling using a UPLC/FLR mass spectrometry platform. http://www.waters.com/webassets/cms/library/docs/ 720003576en.pdf (accessed 13 May 2016).

21 Damen CW, Chen W, Chakraborty $A B$, et al. Electrospray ionization quadrupole ion-mobility time-of-flight mass spectrometry as a tool to distinguish the lot-to-lot heterogeneity in $\mathrm{N}$-glycosylation profile of the therapeutic monoclonal antibody trastuzumab. J Am Soc Mass Spectrom 2009:20:2021-33.

22 Sethuraman N, Stadheim TA. Challenges in therapeutic glycoprotein production. Curr Opin Biotechnol 2006;17:341-6.

23 European Medicines Agency. Avastin, INN-bevacizumab. http://www.ema.europa.eu/ docs/en_GB/document_library/EPAR___Scientific_Discussion/human/000582/ WC500029262.pdf (accessed 13 May 2016).

24 Park El, Manzella SM, Baenziger JU. Rapid clearance of sialylated glycoproteins by the asialoglycoprotein receptor. J Biol Chem 2003:278:4597-602.

25 lida S, Kuni-Kamochi R, Mori K, et al. Two mechanisms of the enhanced antibody-dependent cellular cytotoxicity (ADCC) efficacy of non-fucosylated therapeutic antibodies in human blood. BMC Cancer 2009;9:58.

26 Gorovits B, Krinos-Fiorotti C. Proposed mechanism of off-target toxicity for antibody-drug conjugates driven by mannose receptor uptake. Cancer Immunol Immunother 2013;62:217-23. 